we have

$$
-\int_{C} p d V=\Delta A=-\Delta S \Delta T,
$$

where $C$ is a Carnot cycle which proceeds first along an adiabatic, then along an isothermal, then an adiabatic, and then along an isothermal to the starting point. We conclude that the family of isothermals $T=T_{1}+n \Delta T$ and the family of adiabatics $S=$ $S_{1}+n \Delta S, n=0, \pm 1, \pm 2, \pm 3, \cdots$ drawn in the $p, V$ plane map out equal areas $\Delta A$ in this plane. Thus, if for any gas the isothermals $p=p_{i}(V)$ and the adiabatics $p=p_{a}(V)$ are determined by experiment, the "labels" $S$ and $T$ for the curves may then be determined for it is merely necessary to label as $\mathbf{0}$ the isothermal along which water freezes and as 100 the isothermal along which water boils at atmospheric pressure. The labels of the curves $T=$ const. are then determined by Eq. (3) uniquely. Any pair of curves may be labeled $S=1$ and $S=2$ along any isothermal. The unit of entropy is related to the unit of mechanical energy by resorting again to Eq. (3). The labels for the intervening curves $S=$ const. are also uniquely determined by Eq. (3). Entropy and absolute temperature $S$ and $T$ as thus determined for one gas must be consistent with $S$ and $T$ determined for any other gas due to the principle of conservation of energy. The extent to which the areas $\Delta A$ in the $p, V$ plane are equal indicates the extent to which the postulated equation (2) is valid.

\title{
Correction to my paper
}

\section{ON TRANSFER FUNCTIONS AND TRANSIENTS}

Quarterly of Applied Mathematics, XVI, 273-294 (1958)

By A. H. ZEMANIAN (New York University)

Expression (38) should be replaced by its $(m-n)$ th positive root and the second line below this expression should read, "horizontal line whose ordinate is the $(m-n)$ th positive root of $(m-n)$ !."

The second conclusion of Theorem 8 should be deleted and its proof adjusted such that zeros on the imaginary axis are counted with the zeros in the left half plane (i.e. the symbol $q$ should be discarded and the expression $n-p-q$ should be replaced by $n-p)$. 